\title{
Educazione al counselling per la gestione del Rene Policistico Autosomico Dominante
}

Giornale di Tecniche Nefrologiche e Dialitiche 2018, Vol. 30(2) 125-133

(C) The Author(s) 2018

Article reuse guidelines:

sagepub.com/journals-permissions

DOI: $10.1177 / 0394936218807897$

journals.sagepub.com/home/gtn

\section{(S)AGE}

\section{Aldo Torreggiani' ${ }^{2}$, Emanuela Dorigo' and Luisa Sternfeld Pavia ${ }^{3}$}

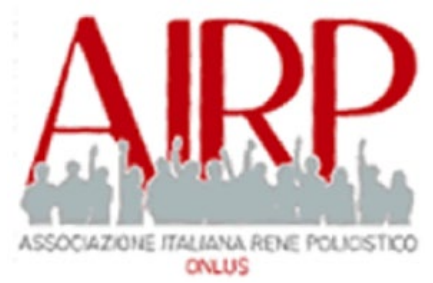

\section{Abstract \\ Counselling education for the Management of autosomal dominant polycystic kidney disease}

By correctly using counselling techniques the nephrology team can face and manage the relationship with patients affected by autosomal dominant polycystic kidney disease (ADPKD), so that patients can consciously prepare to the therapeutic pathway, actively changing their everyday life and improving their own lifestyle.

Counselling is a conscious and strategic way of using information and capabilities to optimally manage the patientdoctor relationship; it is about warm reception, dialogue, communication, support, clear explanation, reassurance, and guidance.

The main objective of counselling is to help patients making decisions that can determine a real change in life, recognizing and getting on with all the efforts to overcome the difficulties, by optimizing the personal, familiar, and cultural resources.

The counsellor abilities help the patient to start speaking about his/her problems by constructing a positive environment in which to feel at ease.

The main tool for building a positive relation is active listening.

The counselling applicability and usefulness in the context of Nephrology is evident: in fact, a trustful relationship between the actors involved (patient-doctor) is the basis of any effective health intervention.

The keys to the therapeutic success are building a deep doctor-patient relationship, creating a better communication with the healthcare team, negotiating the collaboration at every single visit, and timely managing the adverse events.

\section{Keywords}

Counselling, Nephrology, ADPKD, Patient-Doctor relationship

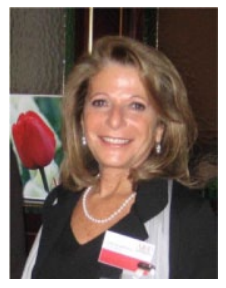

\section{Introduzione}

Il 21 e 22 giugno 2018, al Palazzo delle Stelline a Milano, si è svolto un corso di formazione dal titolo " $C$-time in $A D P K D$ : progetto integrato di educazione al counselling per la gestione del Rene Policistico Autosomico Dominante (ADPKD, autosomal dominant polycystic kidney disease)".

Al corso hanno partecipato diverse equipe di centri di nefrologia di tutta Italia per imparare ad affrontare e gestire, attraverso tecniche di counselling, la relazione con la persona con $\mathrm{ADPKD}$, così da aiutarla a prepararsi consapevolmente ai percorsi di cura, intraprendendo azioni per

\footnotetext{
'Esperto di Manegement, Milano, Italy

${ }^{2}$ Senior Project Manager, T\&C srl

${ }^{3}$ Presidente AIRP onlus, Associazione Italiana Rene Policistico onlus, IT

\section{Corrispondenza:}

Luisa Sternfeld Pavia, Presidente AIRP onlus, Associazione Italiana Rene Policistico onlus, IT.

Email: luisa.sternfeld.airp@renepolicistico.it
} 
cambiare la sua quotidianità e migliorare lo stile di vita. Un'informazione puntuale e la gestione della relazione favoriscono il corretto trattamento della patologia.

Obiettivi del corso sono stati quelli di far acquisire tecniche di counselling utili a gestire il paziente con ADPKD, fornire strumenti a supporto delle azioni di counselling e impostare piani terapeutici appropriati alle caratteristiche del paziente.

Oltre a brevi comunicazioni scientifiche sull'evoluzione della malattia e sui percorsi di cura, la presenza di due attori che hanno interpretato la parte di pazienti ha permesso di condurre un'attività di role play. Tale attività ha coinvolto le equipe di nefrologia in momenti per comunicare la diagnosi, il counselling genetico, eleggibilità e motivazione al trattamento, e counselling sulla necessità di dialisi e trapianto.

L'ADPKD è una patologia ereditaria che colpisce circa 12.5 milioni di persone nel mondo e rappresenta la principale causa genetica di insufficienza renale dell'adulto. ADPKD è una malattia geneticamente eterogenea, ad esordio tardivo. L'età d'insorgenza della malattia, la gravità, e il decorso cli-nico sono condizionati da fattori genetici e ambientali.

È caratterizzata dalla progressiva formazione di cisti renali, che portano all'ingrossamento dell'organo e allo sviluppo di una varietà di sintomi (in particolare ipertensione, dolore, emorragie, ed infezioni) che condizionano in modo estremamente negativo la qualità di vita del paziente.

La funzionalità renale può risultare preservata anche per decenni grazie a meccanismi compensatori, ma quando l'organo è estremamente compromesso, essa declina rapidamente fino ad arrivare alla malattia renale all'ultimo stadio (ESRD, end-stage renal disease) generalmente entro la quarta decade.

Oltre alle manifestazioni renali, l'ADPKD si caratterizza per una serie di complicanze cardiovascolari, epatiche e pancreatiche che peggiorano ulteriormente il quadro clinico del paziente.

Sono stati compiuti molti progressi nella comprensione della patogenesi molecolare e questo ha permesso di definire reali possibilità di trattamento.

Per lungo tempo il percorso di cura si è concentrato sul trattamento della sintomatologia dell'ADPKD, non essendo disponibile nessun trattamento specifico per questa patologia. Tuttavia, un farmaco, il Tolvaptan, ha rappresentato una svolta significativa nella gestione dell'ADPKD, permettendo, in pazienti con caratteristiche definite, di modificare la progressione della malattia e preservare la funzione renale, con la possibilità di migliorare la qualità di vita dei pazienti e ottenere risultati a lungo termine.

Le equipe dei centri di nefrologia devono saper affrontare e gestire, attraverso tecniche di counselling, la relazione con la persona con $\mathrm{ADPKD}$, così da aiutarla $\mathrm{a}$ prepararsi consapevolmente ai percorsi di cura, intraprendendo azioni per cambiare la sua quotidianità e migliorare lo stile di vita.

\section{Che cos'è il counselling}

Il termine inglese counselling deriva dal verbo to counsel che significa "dare consigli".

In realtà, il counselling non intende né fornire sempli-ci consigli né persuadere, piuttosto è inteso come un'attività d'aiuto. Il counselling è una forma di sostegno basata sull'uso della relazione per sviluppare autoconsapevolezza, accettazione delle emozioni e crescita delle risorse personali.

L'O.M.S. definisce il counselling come "processo in cui, attraverso una relazione interpersonale, viene ad istituirsi una comunicazione strutturata tra il consulente e il consultante".

Il counselling è una forma di utilizzo consapevole e strategico delle informazioni e delle abilità necessarie alla gestione ottimale della relazione.

Il counselling comprende attività di accoglienza, dialo-go, comunicazione, supporto, spiegazione, rassicurazione, sostegno e guida.

\section{Chi è il counsellor}

Il counsellor è una persona aperta ed imparziale che attraverso l'ascolto, il supporto e la guida aiuta il paziente a capire i propri bisogni, a superare le crisi e a gestire e risolvere i problemi.

Il counsellor spinge il paziente ad una comprensione di sé e della propria situazione abbastanza profonda così da poter valutare le diverse opzioni disponibili.

\section{Quali sono gli obiettivi del counselling}

Il counselling è orientato alla definizione e soluzione di problemi specifici e a migliorare la capacità di prendere decisioni, affrontare i momenti di crisi, e favorire il confronto con i propri sentimenti e i conflitti interiori.

Lo scopo generale del counselling è aiutare il paziente a prendere decisioni, a promuovere la consapevolezza della necessità di un cambiamento e a riconoscere ed attuare le azioni possibili e utili per superare il problema, ottimizzando le risorse personali, familiari e socio-assistenziali.

Il counselling aiuta, senza giudizi, imposizioni o forzature, ad utilizzare le risorse della persona per cambiare in meglio i suoi comportamenti.

\section{Strategie di counselling}

Informative counselling: $i$ counsellor fornisce informazioni che riguardano argomenti relativi alla malattia e, dopo una fase di ascolto attivo, valuta la necessità di correggere o ampliare le conoscenze già in possesso del paziente, discutendone le implicazioni personali. 
Crisis counselling: le situazioni che possono scatenare una crisi emotiva sono molteplici e legate alla malattia, all'esecuzione di esami diagnostici, alla comparsa di sintomi fisici. In queste circostanze l'obiettivo del counselling è quello di ripristinare il senso di controllo, attraverso alcune tappe che presuppongono lo stare sul "qui ed ora" dei vissuti del soggetto, esplorare $i$ vissuti d'impotenza, disperazione e perdita di controllo, comunicazione $e$ comprensione, chiarire ciò da cui la persona si sente minacciata, fornire supporto e rassicurazione restituendo una valutazione obiettiva della situazione e definendo le stra-tegie che dovranno essere attuate per risolvere la crisi.

Problem-solving counselling: il problema deve essere scomposto in una serie di situazioni più semplici che possono essere affrontate concretamente: la necessità di comunicare il proprio stato, la difficoltà di avere figli, la difficoltà di mantenere la propria autonomia. In queste circostanze, per stimolare un comportamento adattivo, è opportuno seguire alcune tappe che prevedono l'ascoltare attivamente e lasciare spazio all'espressione dei sentimenti, definire il problema, determinare precisamente uno o più obiettivi da raggiungere, individuare e selezionare strategie che permettano il raggiungimento degli obiettivi, rendere operative le strategie e valutare gli obiettivi raggiunti.

Decision making counselling: l'obiettivo è in questo caso condurre la persona, attraverso un'accurata analisi delle circostanze, a una scelta razionale, adattiva, il più possibile vantaggiosa e rispondente ai suoi bisogni e desideri.

I passi da compiere sono: identificare con precisione l'obiettivo da raggiungere, aiutare la persona a ipotizzare il maggior numero possibile di alternative disponibili, compiere un'analisi accurata, aiutare la persona a esprime-re la sua scelta, favorire un'analisi obiettiva delle implicazioni, programmare un piano operativo.

\section{Abilità rischieste per un buon counselling}

Le abilità del counsellor danno la possibilità al paziente di iniziare a parlare delle sue problematiche creando un ambiente positivo in cui possa sentirsi a proprio agio.

Lo strumento fondamentale per la costruzione di una relazione d'aiuto è l'ascolto attivo che rappresenta una capacità complessa ricettiva e attiva, comprendente altre specifiche abilità.

Le abilità richieste per un buon counselling sono:

Prestare attenzione, cioè manifestare la propria attenzione attraverso una postura aperta, ovvero un atteggiamento che comunichi disponibilità.

Osservare, cioè focalizzare la propria attenzione sulla comunicazione non verbale e sulle eventuali incongruenze con quanto detto.

Ascoltare, che prevede una predisposizione all'interesse per i problemi del paziente ed una concentrazione su quanto viene detto.
Essere empatici, che rappresenta lo strumento finalizzato alla costruzione della relazione di fiducia, di cono-scenza e di comprensione.

Accettare incondizionatamente, che ci permette di non rifiutare sentimenti, esperienze e convinzioni diverse dalle nostre manifestando una sincera disponibilità.

Essere coerenti, che permette di stabilire un rapporto di fiducia che dev'essere acquisita tramite una crescente consapevolezza dei filtri attraverso i quali passano le informazioni e che sono essenzialmente la cultura, i valori personali, i problemi personali e lo stato emotivo che possono influenzare lo svolgimento del counselling.

\section{Dove, come e quando condurre un colloquio di counselling}

La forma di collaborazione tra il counsellor ed il paziente deve essere svolto in tempi predefiniti e concordati, previa individuazione di spazi che permettano un'adeguata comunicazione in intimità e garantiscano riservatezza. Lo spazio fisico e temporale si definisce "setting" ed idealmente dovrebbe prevedere una stanza priva di distrazioni, arredata in modo semplice ma con colori caldi; il colloquio dovrebbe avvenire tra due persone sedute alla stessa altezza, la fonte di luce dovrebbe garantire la lettura delle espressioni del volto e della postura per facilitare la comunicazione non verbale.

Il setting interno rappresenta la condizione propria del counsellor di apertura verso quella persona in quel momento, la sua capacità di ascoltare senza giudicare e senza sorprendersi.

Il supporto emotivo è necessario per ridurre l'ansia, facilitare l'espressione e la discussione dei sentimenti vissuti, ridurre lo stress e la depressione, restituire alla persona un senso di controllo sulla situazione.

Il linguaggio dovrà essere conforme a quello utilizzato dalla persona, dal suo livello d'istruzione, le sue conoscenze specifiche, il contesto culturale, i suoi valori ed il suo stato psicoemotivo.

\section{Suggerimenti su come condurre un colloquio di counselling}

\section{Prima di parlare è bene ascoltare}

È importante saper ascoltare per ricevere informazioni tramite l'udito, per dare significato a tali informazioni, per decidere cosa pensare o sentire in merito alle informazioni ricevute e infine per rispondere a ciò che si è udito. Per essere un perfetto ascoltatore è necessario innanzitutto mettere l'interlocutore a proprio agio, non parlare di sé se non dopo aver fatto parlare l'altro, evitare le distrazioni, cercare di essere pazienti, non perdere le staffe e sospendere qualsiasi giudizio di valore come "è giusto, è sbagliato". Per essere un perfetto ascoltatore è necessario ascoltare con tutto il corpo assumendo un atteggiamento positivo e 
incoraggiante, con una postura frontale rispetto all'interlocutore, dando il proprio assenso con il capo ad ogni frase e mantenendo sempre il contatto visivo.

\section{Ricercare le sintonie}

Individuare i punti di contatto significa creare un presupposto di "confidenza" che, adeguatamente sviluppato, porterà fiducia reciproca nel lungo periodo. Attraverso la conoscenza della porta percettiva d'ingresso prevalente del mio interlocutore (visivo, uditivo, cinestesico) potrò creare similarità riproponendo linguaggi simili a quelli utilizzati dal mio interlocutore. Quello che a noi serve è una lettura veloce della porta preferita dall'interlocutore per stabilire un rapporto ottimale di comunicazione, nonché per la scelta della terminologia convincente e di un linguaggio non verbale e paraverbale coerente.

$\mathrm{Al}$ counsellor è richiesto un ascolto attento e un adattamento progressivo allo stile comunicazionale di chi gli è di fronte.

Deve utilizzare tutti i suoi sensi per carpire al meglio i messaggi che l'altra persona invia.

\section{Usare tutti i canali della comunicazione}

La comunicazione non è solo il linguaggio verbale che usia-mo ma anche l'atteggiamento e il modo che l'accompagna. Il linguaggio verbale trasmette il contenuto della comunica-zione attraverso parole, concetti e simboli. Il linguaggio paraverbale definisce la modalità di comunicazione attraverso il tono, il ritmo, la velocità della voce. Il linguaggio non verbale definisce la relazione che caratterizza la comunicazione attraverso i gesti, la postura, le espressioni del viso, il movimento, lo sguardo.

\section{Counselling ed educazione del paziente facilitano il successo terapeutico}

L'applicabilità e l'utilità del counselling nel contesto della nefrologia appare evidente. La relazione di fiducia tra gli attori del progetto (medico-paziente) è alla base di qualsiasi efficace intervento sanitario.

Costruire un'intensa relazione medico-paziente, migliorare la comunicazione con il team sanitario, rinegoziare l'aderenza ad ogni visita clinica e gestire gli eventi avversi in anticipo sono la chiave per il successo terapeutico.

\section{Dichiarazione di assenza di conflitto di interessi}

Gli Autori dichiarano di non avere conflitti di interessi.

\section{Finanziamenti}

Gli Autori dichiarano di non aver ricevuto finanziamenti specifici da qualsiasi ente nei settori pubblico, privato o senza fini di lucro. 


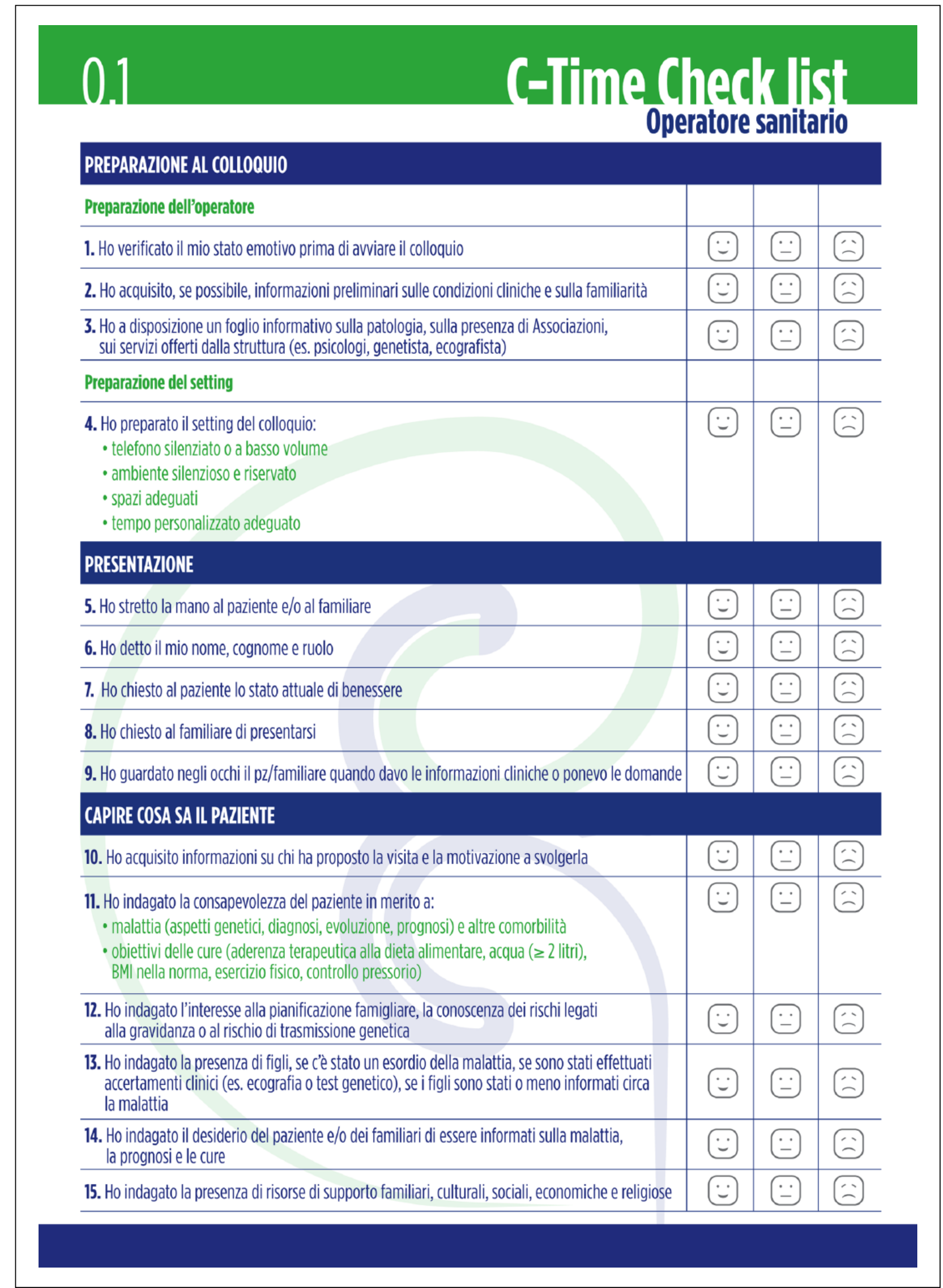




\section{CONDIVIDERE LE INFORMAZIONI}

16. Ho fornito informazioni rispetto al significato delle cure e gli obiettivi, nel dettaglio:

- quali sono le possibili cure

- a chi si rivolgono

- il percorso clinico-assistenziale

- la necessità di ulteriori accertamenti

17. (se prescrivibile) assunzione Tolvaptan (caratteristiche del farmaco, modalità e tempi di assunzione, benefici, motivazione all'aderenza, effetti collaterali)

18. Ho dato spazio alle domande del paziente e/o dei familiari

19. Ho dato risposte a tutte le richieste del paziente

20. Mi sono accertato che abbiano compreso (anche facendo ripetere)

\section{DARE SPAZIO ALLE EMOZIONI}

\begin{tabular}{|c|c|c|c|}
\hline 21. Ho risposto in modo empatico & $\because$ & $\because$ & 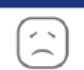 \\
\hline 22. Ho rassicurato il paziente laddove ve ne era la possibilità & $\because$ & $\because$ & 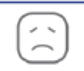 \\
\hline 23. Ho sfatato false credenze/paure sulla malattia o la terapia & $\because$ & & 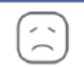 \\
\hline 24. Ho stimolato la motivazione al progetto di cura & $\because$ & 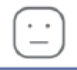 & 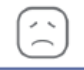 \\
\hline $\begin{array}{l}\text { 25. Ho rilevato emozioni di difficile gestione per il paziente (es. ansia etc...) e ho suggerito } \\
\text { un supporto psicologico (psicologo, associazione paziente, gruppi di ascolto /aiuto) }\end{array}$ & & & 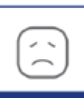 \\
\hline \multicolumn{4}{|l|}{ PIANIFICARE E RIASSUMERE } \\
\hline 26. Ho condiviso il progetto di cura & $\because$ & 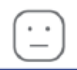 & 气 \\
\hline $\begin{array}{l}\text { 27. Ho dato disponibilità a ulteriori momenti di confronto, lasciando eventuali contatti telefonici } \\
\text { di riferimento }\end{array}$ & $\curvearrowright$ & 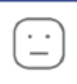 & $\approx$ \\
\hline 28. Ho effettuato una sintesi delle informazioni verificando che non ci siano stati fraintendimenti & $\because$ & $\because$ & $\Theta$ \\
\hline $\begin{array}{l}\text { 29. Ho verificato il mio stato emotivo al termine del colloquio prima di riprendere l'attività } \\
\text { o prima di sostenere un nuovo colloquio }\end{array}$ & $\Theta$ & 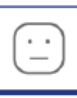 & $\Xi$ \\
\hline
\end{tabular}


$0.2 \quad$ G - Time Check list

Operatore sanitario

Avvio al trattamento con tolvaptan

\section{CONDIVIDERE LE INFORMAZIONI}

1. Ho spiegato al paziente i benefici del farmaco (funzione renale, volume renale) sottolineando il vantaggio del ritardo dell'ingresso in dialisi

2. Ho fornito suggerimenti pratici rispetto alle modalità di assunzione

- iniziare l'uso del farmaco in giornate festive cosi da capire e registrare le reazioni del proprio corpo

- puntare la sveglia la mattina presto (tra le 6 e le 7), assumere il farmaco, provvedere alle cure personali e terminare con la colazione

- controllare il tempo alla prima minzione per organizzare la giornata

- la seconda dose assunta nel primo pomeriggio avrà meno impatto sulla nicturia

3. Ho spiegato come garantire un corretto apporto idrico con semplici accorgimenti:

- acqua sempre a disposizione in ufficio, in borsa, in viaggio

- nell'incertezza bere un bicchiere d'acqua ad ogni minzione, avendo cura di cambiare tipo di acqua

- bere 102 bicchieri di liquidi prima di andare a letto a prescindere dalla sete percepita

- assecondare il senso di sete

4. Ho spiegato che con l'assunzione di tolvaptan si può arrivare ad urinare da 3 fino a 8 litri al giorno

5. Ho spiegato che nei primi tre mesi di terapia, bisogna prendere le misure; è necessario fare controlli periodici; dopo questa fase di adattamento, il percorso diventa più semplice

6. Ho spiegato al paziente come riconoscere i sintomi della disidratazione (attenzione in caso di dissenteria e vomito) e come valutare il bilancio idrico

7. Mi sono accertato che il paziente e/o caregiver abbiano compreso (anche facendo ripetere)

8. Ho consegnato l'informativa per il MMG 


\section{Gime Chead list}

Paziente

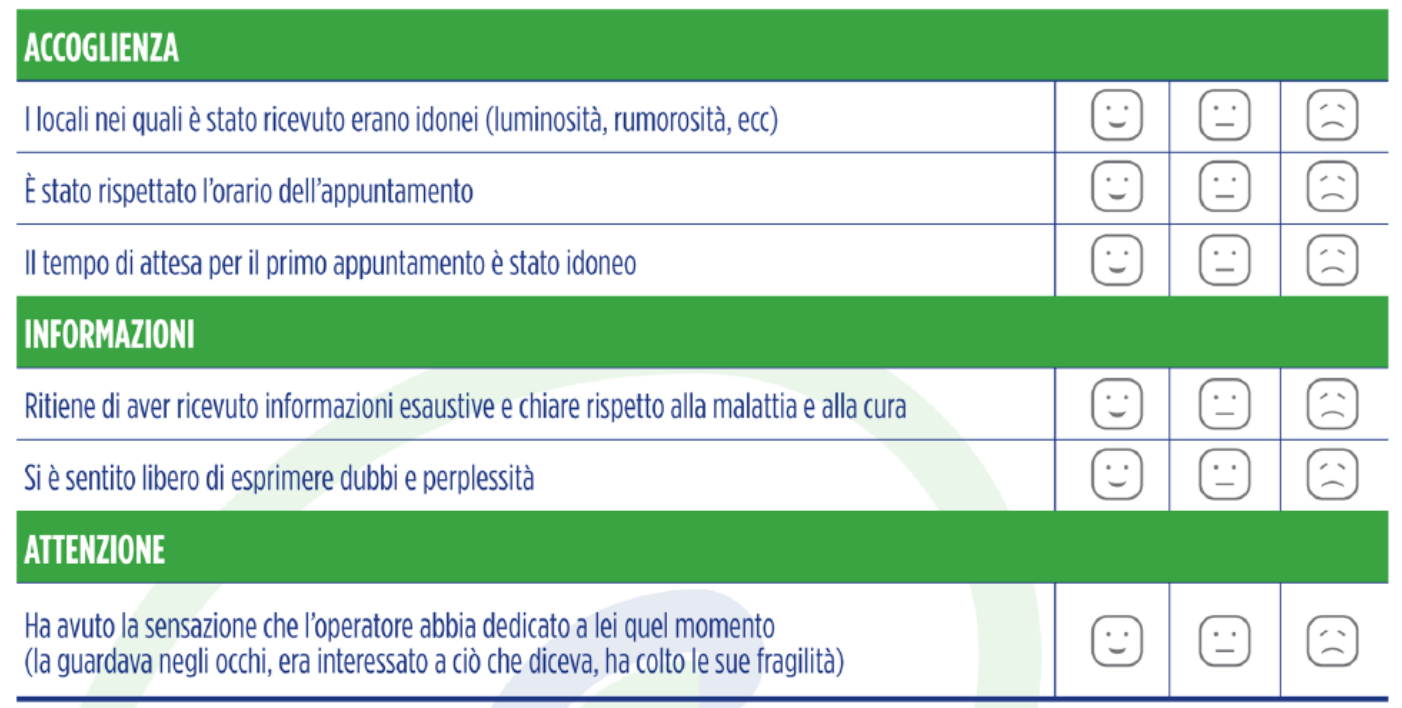




\section{2 \\ GTime Chedk list \\ Paziente \\ Avvio al trattamento con tolvaptan}

\begin{tabular}{|c|c|c|c|}
\hline Conosce i benefici che la terapia avrà sulla progressione della sua malattia? & $\ddot{\theta}$ & $\because$ & E \\
\hline Ha avuto indicazioni sulla posologia del farmaco ed eventuali effetti collaterali? & $\Theta$ & $\because$ & E \\
\hline Ha ricevuto consigli pratici per le modalità di assunzione di tolvaptan? & $\because$ & $\because$ & E \\
\hline Ha ricevuto tutte le indicazioni per garantire un adeguato apporto idrico? & $\theta$ & $\because$ & 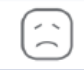 \\
\hline Ha ricevuto informazioni chiare sui controlli periodici da effettuare? & $\theta$ & $\because$ & 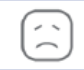 \\
\hline $\begin{array}{l}\text { Ha avuto i contatti telefonici da utilizzare in caso di emergenza o per avere ulteriori } \\
\text { chiarimenti durante il trattamento? }\end{array}$ & 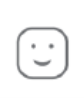 & 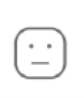 & 田 \\
\hline Ha ricevuto l'informativa per il suo Medico di Medicina Generale? & $\because$ & 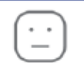 & $\Theta$ \\
\hline
\end{tabular}

